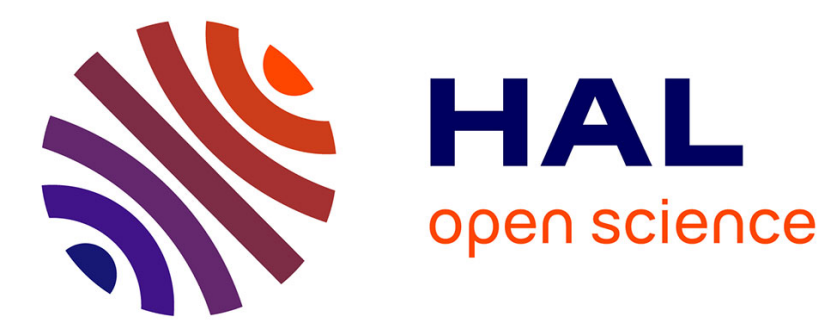

\title{
A Transfer matrix-perturbation approach to the dynamics of chains of nonlinear sliding beams
}

\author{
Angelo Luongo, Francesco Romeo
}

\section{To cite this version:}

Angelo Luongo, Francesco Romeo. A Transfer matrix-perturbation approach to the dynamics of chains of nonlinear sliding beams. Journal of Vibration and Acoustics, 2006, 128 (2), pp.190-196. hal-00790182

\section{HAL Id: hal-00790182 \\ https://hal.science/hal-00790182}

Submitted on 19 Feb 2013

HAL is a multi-disciplinary open access archive for the deposit and dissemination of scientific research documents, whether they are published or not. The documents may come from teaching and research institutions in France or abroad, or from public or private research centers.
L'archive ouverte pluridisciplinaire HAL, est destinée au dépôt et à la diffusion de documents scientifiques de niveau recherche, publiés ou non, émanant des établissements d'enseignement et de recherche français ou étrangers, des laboratoires publics ou privés. 


\section{A Transfer-Matrix-Perturbation Approach to the Dynamics of Chains of Nonlinear Sliding Beams}

67040 Monteluco di Roio (L'Aquila), Italy e-mail: luongo@ing.univaq.it

\section{Francesco Romeo ${ }^{1}$ \\ Assistant Professor \\ Dipartimento di Ingegneria \\ Strutturale e Geotecnica, \\ Università di Roma "La Sapienza," \\ Via Gramsci 53, 00197 Rome, Italy e-mail: francesco.romeo@uniroma1.it}

\begin{abstract}
Chains of nonlinear shear indeformable beams with distributed mass, resting on movable supports, are considered. To determine the dynamic response of the system, the transfermatrix approach is merged with the harmonic balance method and a perturbation method, thereby transforming the original space-temporal continuous problem into a discrete one-dimensional map $\mathbf{x}_{k+1}=\mathrm{F}\left(\mathbf{x}_{k}\right)$ expressed in terms of the state variables $\mathbf{x}_{k}$ at the interface between adjacent beams. Such transformation does not imply any discretization, because it is obtained by integrating the single-element field equations. The state variables consist of both first-order variables, namely, transversal displacement and couples, and second-order variables, which are longitudinal displacement and axial forces. Therefore, while the linear problem is monocoupled, the nonlinear one becomes multicoupled. The procedure is applied to determine frequency-response relationship under free and forced vibrations.
\end{abstract}

\section{Introduction}

The transfer matrix method has been widely used to analyze the dynamics of linear periodic systems $[1,2]$. In this realm, analytical, design, and computational aspects have been recently investigated by the authors, respectively, in [3-5]. Although its applicability is restricted, due to numerical problems, to a limited number of periodic units, the method allows one to reduce the dimension of the problem to the number of degrees of freedom of the single element.

It is well known that the disturbance propagation through multicoupled linear periodic structures is governed by the frequencydependent transfer-matrix eigenvalues. On the frequency axis, there exist intervals or bands where disturbances propagate harmonically without attenuation (pass bands), in which the eigenvalues are complex with unitary modulus, and bands where the disturbances decay (stop bands), in which the eigenvalues are real and different from 1. As soon as the coupling coordinates are more than 1 , there exist further frequency bands characterized by disturbance harmonic propagation with attenuation (complex bands) where pairs of complex conjugate eigenvalues, with modulus different from 1, exist. Moreover, the natural frequencies fall within the pass bands.

In contrast to the vast literature concerning linear periodic structures, few works have thus far appeared in the mechanical context on the dynamics of nonlinear periodic systems. Monocoupled periodic systems of infinite extent with material nonlinearities have been addressed in [6]. Two different asymptotic approaches have been devised for studying standing (stop-band) and traveling (pass-band) waves; amplitude-dependent frequencies bounding nonlinear propagation and attenuation zones have been found. In [7], an array of elastic oscillators coupled through buckling sensitive elastica has been addressed both numerically and experimentally. The existence of transition from solitonlike motions to spatially and temporally disordered motions due to a sudden excitation has been shown relying on a modified Toda lattice

\footnotetext{
${ }^{1}$ Corresponding author
}

model. An extension of the transfer-matrix approach to postbuckling problems of periodic systems has been proposed in [8].

In this work, the latter approach is further developed to analyze free and forced dynamics of chains of multicoupled nonlinear beams. In Sec. 2, the nonlinear continuous-beam model is derived. Afterward, in Sec. 3, the nonlinear element transfer function is derived through a perturbation method. By iterating the nonlinear element map, a consistent system transfer function is obtained in Sec. 4. Free and forced oscillations are studied in Sec. 5, and the relevant numerical results are eventually shown in Sec. 6 .

\section{Continuous Beam on Sliding Supports}

A chain of $N$ shear indeformable beams with distributed mass $m$, resting on movable supports, as schematically depicted in Fig. 1 , is considered. The single beam, of length $l$ and flexural stiffness $E I$, is also axially indeformable. The displacement field is defined by the longitudinal and transverse components $u(s)$ and $v(s)$, respectively, and by a rotation $\varphi(s)$ of the beam cross section, $s$ being the abscissa. The conditions of zero transverse shear and elongation, imply, respectively,

$$
\sin \varphi=v^{\prime} ; \quad \epsilon:=\sqrt{\left(\left(1+u^{\prime}\right)^{2}+v^{\prime 2}\right)}-1=0
$$

from which the curvature $\kappa$ follows:

$$
\kappa:=\varphi^{\prime}=\frac{v^{\prime \prime}}{\sqrt{1-v^{\prime 2}}}
$$

The equations of motion are derived from the stationarity of the constrained Hamiltonian

$$
\begin{aligned}
\bar{H}= & \int_{t_{1}}^{t_{2}} \int_{0}^{l}\left[\frac{1}{2} m\left(\dot{u}^{2}+\dot{v}^{2}\right)-\frac{1}{2} E I \kappa^{2}-\lambda \epsilon\right] d s d t+g_{L} u_{L}+m_{L} \varphi_{L} \\
& +g_{R} u_{R}+m_{R} \varphi_{R}
\end{aligned}
$$

where $u_{H}, \varphi_{H}, g_{H}, m_{H},(H=L, R)$ are the end displacements and forces and the Lagrangian parameter $\lambda(s)$ has been introduced. By considering the series expansions, $\kappa \simeq v^{\prime \prime}\left[1+\left(v^{\prime 2} / 2\right)\right], \quad \epsilon \simeq u^{\prime}$ $+\frac{1}{2} v^{\prime 2}$, the stationarity condition leads to the set of partial differential equations

$$
v^{\prime \prime \prime \prime}+\beta^{4} \ddot{v}+\left[v^{\prime}\left(v^{\prime} v^{\prime \prime}\right)^{\prime}\right]^{\prime}-\left(\lambda v^{\prime}\right)^{\prime}=0
$$




\begin{tabular}{|c|c|c|c|}
\hline$\infty$ & ac & 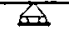 & $\bar{m}$ \\
\hline$k-1$ & $k$ & $k+1$ & $k+2$ \\
\hline
\end{tabular}

(a)

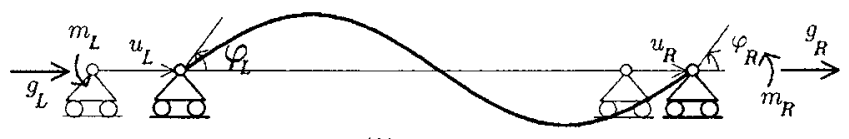

(b)

Fig. 1 (a) Chain of nonlinear beams and (b) inextensible and shear indeformable single beam

$$
\begin{gathered}
\beta^{4} \ddot{u}-\lambda^{\prime}=0 \\
u^{\prime}+\frac{1}{2} v^{\prime 2}=0
\end{gathered}
$$

where the following nondimensional quantities have been used:

$$
\begin{gathered}
\bar{v}=\frac{v}{l}, \quad \bar{u}=\frac{u}{l}, \quad \bar{s}=\frac{s}{l}, \quad \tau=\omega t, \quad \beta^{4}=\frac{m \omega^{2}}{E I} l^{4}, \quad \bar{\lambda}=\frac{\lambda l^{2}}{E I} \\
\bar{m}=\frac{m l}{E I}, \quad(\cdot)=\frac{d}{d \tau}
\end{gathered}
$$

where $\omega$ represents a frequency to be specified later and the overbars have been omitted. By prescribing the force $g_{R}$ and all the displacements except $u_{R}$, the boundary conditions follow:

$$
\begin{gathered}
v(0, \tau)=0 \\
v^{\prime}(0, \tau)+\frac{1}{6} v^{\prime 3}(0, \tau)=\varphi_{L} \\
v(1, \tau)=0 \\
v^{\prime}(1, \tau)+\frac{1}{6} v^{\prime 3}(1, \tau)=\varphi_{R} \\
u(0, \tau)=u_{L} \\
\lambda(1, \tau)=g_{R}
\end{gathered}
$$

where the series expansion $\varphi \simeq v^{\prime}+\frac{1}{6} v^{\prime 3}$ has been used. Having solved the boundary value problem (4) and (6), by using the moment-curvature relation, the unknown variables at both ends are obtained

$$
m_{L}=-\left[v^{\prime \prime}+\frac{1}{2} v^{\prime \prime} v^{\prime 2}\right]_{0, \tau}
$$

$$
\begin{gathered}
m_{R}=\left[v^{\prime \prime}+\frac{1}{2} v^{\prime \prime} v^{\prime 2}\right]_{1, \tau} \\
u_{R}=u(1, \tau) \\
g_{L}=-\lambda(0, \tau)
\end{gathered}
$$

In view of the transfer-matrix formulation, it is convenient to parametrize the solution in terms of the left-hand variables $\left(\varphi_{L}, m_{L}, g_{L}, u_{L}\right)$; therefore, Eqs. $(6 d)$ and $(6 f)$ are replaced by Eqs. $(7 a)$ and $(7 d)$.

\section{Nonlinear Element Transfer Function}

To solve the nonlinear problem (4), with the proper boundary conditions (6) and (7), use is made of the harmonic balance method. By retaining only the first harmonic in $v(s, \tau)$, Eqs. $(4 c)$ and $(4 b)$ ordinately provide with the time dependence of $u(s, \tau)$ and $\lambda(s, \tau)$, namely,

$$
v(s, \tau)=\hat{v}(s) \cos \tau, \quad u(s, \tau)=\hat{u}(s) \cos ^{2} \tau, \quad \lambda(s, \tau)=\hat{\lambda}(s) \cos 2 \tau
$$

Consequently, $\omega$ in Eq. (5) is the frequency of the (prevailing) transversal motion. The space-dependent functions $\hat{v}(s), \hat{u}(s)$, and $\hat{\lambda}(s)$ are then expanded in series of an artificially introduced small bookkeeping parameter $\varepsilon$, having the meaning of transversal motion amplitude, i.e., $\varepsilon=(O\|\hat{v}(s)\|)$. Aiming at considering only significant (non-vanishing) quantities, incomplete series are adopted, according to the following considerations. If Eq. (4) admit the solution $(v, u, \lambda)$, they also admit the solution $(-v, u, \lambda)$, which describes a state of the beam symmetrical with respect to its undeformed axis. Therefore, by changing the sign of the amplitude $\varepsilon, v$ must change sign (i.e., it is an odd function of $\varepsilon$ ), whereas $u$ and $\lambda$ must keep their sign (i.e., they are even functions of $\varepsilon$ ). By also taking into account Eq. (6) and (7), the following unknowns series expansions and ordering of the boundary terms are introduced

$$
\begin{gathered}
\hat{v}=\varepsilon v_{1}+\varepsilon^{3} v_{3}+\ldots, \quad \hat{u}=\varepsilon^{2} u_{2}+\ldots, \quad \hat{\lambda}=\varepsilon^{2} \lambda_{2}+\ldots \\
\varphi_{H}=\varepsilon \widetilde{\varphi}_{H}, \quad u_{H}=\varepsilon^{2} \widetilde{u}_{H}, \quad g_{H}=\varepsilon^{2} \widetilde{g}_{H}(H=L, R)
\end{gathered}
$$

From Eq. (9) it turns out that the longitudinal displacement $u$ and the axial force $\lambda$ are second-order variables with respect to the transversal displacement $v$. They allow the kinematical inextensibility condition ( $4 c$ ) and the equilibrium in the longitudinal direction $(4 b)$ to be satisfied in the nonlinear range.

By substituting Eqs. (9) into Eq. (4), Eqs. (6a)-(6c), (6e), (7a), and $(7 d)$, and by omitting hat and tilde, the perturbation equations follow

$$
\begin{aligned}
& \epsilon: v_{1}^{\prime \prime \prime \prime}-\beta^{4} v_{1}=0 \\
& \epsilon^{2}: u_{2}^{\prime}+\frac{1}{2} v_{1}^{\prime 2}=0 \\
& \lambda_{2}^{\prime}-2 \beta^{4} u_{2}=0
\end{aligned}
$$

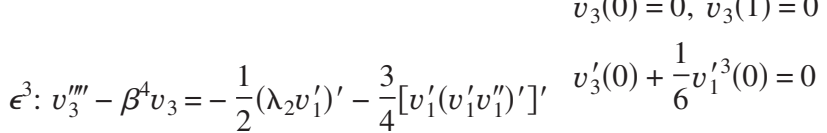

$$
\begin{aligned}
& v_{3}^{\prime \prime}(0)+\frac{1}{2} v_{1}^{\prime 2}(0) v_{1}^{\prime \prime}(0)=0
\end{aligned}
$$




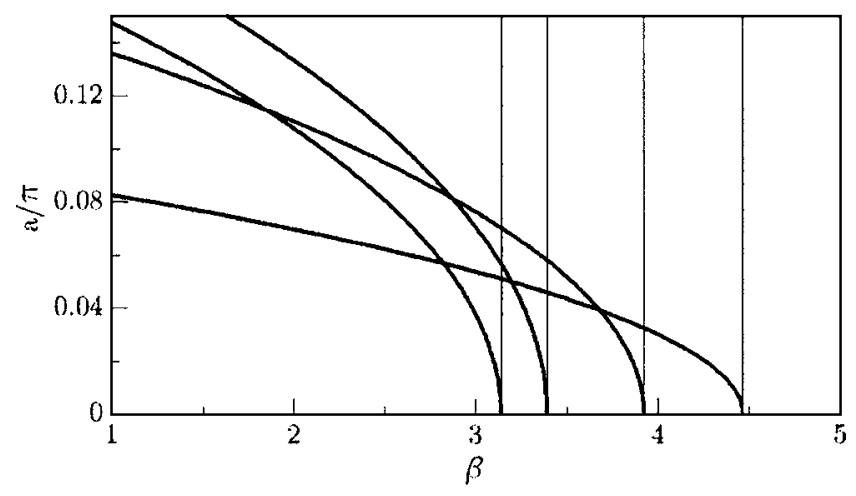

Fig. 2 First four natural frequencies-amplitude relationship for a four four-bay beam: thick lines, nonlinear; thin lines, linear

By solving Eq. (10) according to the increasing powers of $\varepsilon$, the corresponding solutions are obtained in the form

$$
\begin{aligned}
& \epsilon: v_{1}(s)=\varphi_{L} F_{\varphi}(s)+m_{L} F_{m}(s) \\
& u_{2}(s)=u_{2}(0)-\frac{1}{2} \int_{0}^{s} v_{1}^{\prime 2} d s=u_{L}+\varphi_{L}^{2} F_{\varphi \varphi}(s)+m_{L}^{2} F_{m m}(s) \\
& +\varphi_{L} m_{L} F_{\varphi m}(s) \\
& \epsilon^{2}: \lambda_{2}(s)=\lambda_{2}(0)+2 \beta^{4} \int_{0}^{s} u_{2} d s= \\
& -g_{L}+2 \beta^{4}\left[u_{L} s+\varphi_{L}^{2} G_{\varphi \varphi}(s)+m_{L}^{2} G_{m m}(s)+\varphi_{L} m_{L} G_{\varphi m}(s)\right] \\
& \epsilon^{3}: v_{3}(s)=\varphi_{L}^{3} F_{\varphi \varphi \varphi}(s)+\varphi_{L}^{2} m_{L} F_{\varphi \varphi m}(s)+\varphi_{L} m_{L}^{2} F_{\varphi m m}(s) \\
& +m_{L}^{3} F_{m m m}(s) \\
& +g_{L}\left[\varphi_{L} H_{\varphi}(s)+m_{L} H_{m}(s)\right]+u_{L}\left[\varphi_{L} K_{\varphi}(s)+m_{L} K_{m}(s)\right]
\end{aligned}
$$

where $F_{\alpha}, F_{\alpha \alpha}, F_{\alpha \alpha \alpha}, G_{\alpha \alpha}, H_{\alpha}$, and $K_{\alpha}$, with $\alpha=\varphi, m$, are combinations of circular, hyperbolic, and polynomial functions. As expected, $v_{1}(s)$ and $v_{3}(s)$ are linear and cubic, respectively, with respect to $\varphi_{L}, m_{L}$. Moreover $v_{3}(s)$ is bilinear in the products of $\varphi_{L}$ and $m_{L}$, with the second-order variables $u_{L}, g_{L}$.

Given the perturbative expansions, the remaining Eqs. (6d), $(6 f),(7 b)$, and $(7 c)$ are rewritten as

$$
\varepsilon \varphi_{R}=\varepsilon v_{1}^{\prime}(1)+\varepsilon^{3}\left[v_{3}^{\prime}(1)+\frac{1}{6} v_{1}^{\prime 3}(1)\right]
$$

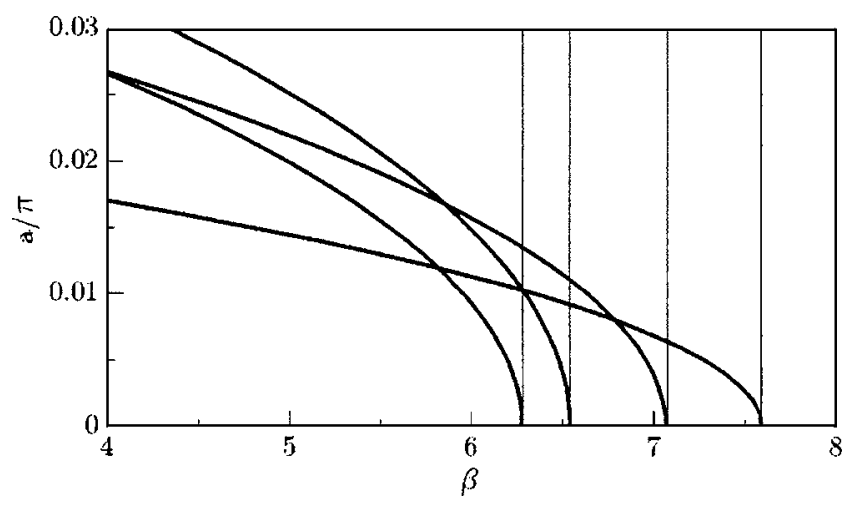

Fig. 3 Second four natural frequencies-amplitude relationship for a four-bay beam: thick lines, nonlinear; thin lines, linear

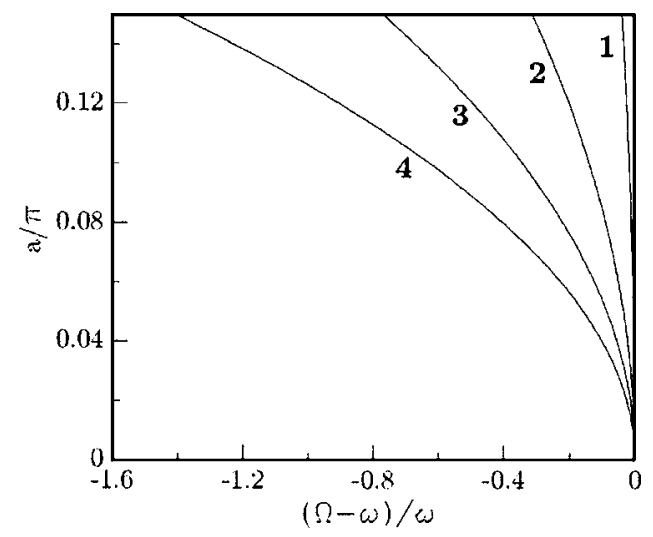

Fig. 4 First natural frequencies-amplitude relationship for a chain with number of beams increasing from 1 to $4(\Omega, \omega$ : nonlinear and linear frequency)

$$
\begin{gathered}
\varepsilon m_{R}=-\varepsilon v_{1}^{\prime \prime}(1)-\varepsilon^{3}\left[v_{3}^{\prime \prime}(1)+\frac{1}{2} v_{1}^{\prime \prime}(1) v_{1}^{\prime 2}(1)\right] \\
\varepsilon^{2} u_{R}=\varepsilon^{2} u_{2}(1) \\
\varepsilon^{2} g_{R}=-\varepsilon^{2} \lambda_{2}(1)
\end{gathered}
$$

They represent the nonlinear relationship between right and lefthand variables. Continuity and equilibrium at the generic node $k$ imply $d_{R, k}=d_{L, k+1} \doteq d_{k}(d=\varphi, u)$ and $f_{R, k}=-f_{L, k+1} \doteq f_{k}(f=m, g)$, respectively, where the second index labels the element. By substituting in Eqs. (12) $v_{1}(s), v_{3}(s), u_{2}(s)$ and $\lambda_{2}(s)$, as given by Eq. (11), the element transfer function, linking the state variables at adjacent interfaces, is obtained

$$
\begin{gathered}
\varepsilon\left(\begin{array}{c}
\varphi \\
m
\end{array}\right)_{k+1}=\varepsilon\left[\begin{array}{ll}
t_{d d} & t_{d f} \\
t_{f d} & t_{f f}
\end{array}\right]\left(\begin{array}{c}
\varphi \\
m
\end{array}\right)_{k}+\varepsilon^{3}\left(\begin{array}{l}
\mathcal{T}_{\varphi}\left(\varphi_{k}, m_{k}, u_{k}, g_{k}\right) \\
\mathcal{T}_{m}\left(\varphi_{k}, m_{k}, u_{k}, g_{k}\right)
\end{array}\right) \\
\varepsilon^{2}\left(\begin{array}{l}
u \\
g
\end{array}\right)_{k+1}=\varepsilon^{2}\left[\begin{array}{cc}
1 & 0 \\
-2 \beta^{4} & 1
\end{array}\right]\left(\begin{array}{l}
u \\
g
\end{array}\right)_{k}+\varepsilon^{2}\left(\begin{array}{l}
\mathcal{T}_{u}\left(\varphi_{k}, m_{k}\right) \\
\mathcal{T}_{g}\left(\varphi_{k}, m_{k}\right)
\end{array}\right)
\end{gathered}
$$

where $t_{p q},(p, q)=(d, f)$, are the usual entries of the monocoupled linear transfer matrix [9] and $\mathcal{T}_{\alpha},(\alpha=\varphi, m, u, g)$, are the components of the nonlinear part of the element transfer function.

\section{Nonlinear System Transfer Function}

In order to link the state variables at the end of the chain, the map ((13) and (14)) must be iterated consistently with the approximation order. Toward this goal, the discrete counterpart of the perturbation scheme adopted for the continuous problem is followed. By setting $\mathbf{v}=(\varphi, m)$ and $\mathbf{u}=(u, g)$, after reabsorbing $\varepsilon$, the Eqs. (13) and (14) are rewritten as

$$
\begin{gathered}
\mathbf{v}(k+1)-\mathbf{T}_{v} \mathbf{v}(k)=\mathcal{T}_{v}[\mathbf{v}(k), \mathbf{u}(k)] \\
\mathbf{u}(k+1)-\mathbf{T}_{u} \mathbf{u}(k)=\mathcal{T}_{u}[\mathbf{v}(k)]
\end{gathered}
$$

By defining $\mathbf{T}_{u_{0}}=\mathbf{T}_{u}\left(\beta_{0}\right)$ and $\mathbf{T}_{v_{0}}=\mathbf{T}_{v}\left(\beta_{0}\right)$ and performing the following series expansions in analogy to Eq. (9):

$$
\begin{gathered}
\mathbf{v}=\epsilon \mathbf{v}_{1}+\epsilon^{3} \mathbf{v}_{3} \quad \mathbf{u}=\epsilon^{2} \mathbf{u}_{2} \quad \beta=\beta_{0}+\epsilon^{2} \beta_{2} \\
\mathbf{T}_{\alpha}=\mathbf{T}_{\alpha 0}+\epsilon^{2} \beta_{2} \mathbf{T}_{\alpha 0}^{\prime} \quad \text { with } \alpha=u, v
\end{gathered}
$$



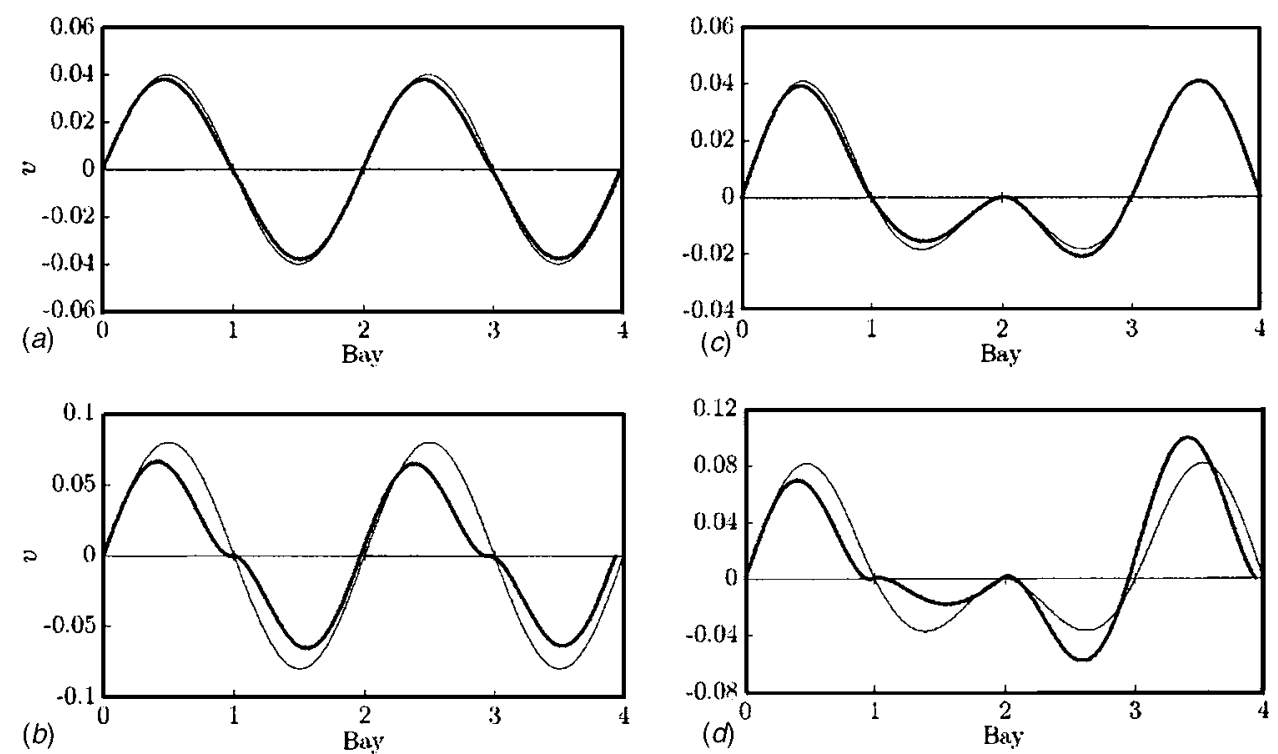

Fig. 5 First and second mode of a four-bay beam: (a) first mode $(\beta=\pi) a=0.04 \pi,(b)$ first mode $(\beta=\pi) \quad a=0.08 \pi,(c)$ second mode $(\beta=3.393) a=0.04 \pi$, and $(d)$ second mode $(\beta=3.393) a$ $=0.08 \pi$; thick lines, nonlinear; thin lines, linear

the following perturbation equations are obtained:

$$
\begin{gathered}
\boldsymbol{\epsilon}: \mathbf{v}_{1}(k+1)-\mathbf{T}_{v 0} \mathbf{v}_{1}(k)=0 \\
\epsilon^{2}: \mathbf{u}_{2}(k+1)-\mathbf{T}_{u_{0}} \mathbf{u}_{2}(k)=\mathcal{T}_{u}\left[\mathbf{v}_{1}(k)\right] \\
\epsilon^{3}: \mathbf{v}_{3}(k+1)-\mathbf{T}_{v_{0}} \mathbf{v}_{3}(k)=\beta_{2} \mathbf{T}_{v_{0}}^{\prime} \mathbf{v}_{1}(k)+\mathcal{T}_{v}\left[\mathbf{v}_{1}(k), \mathbf{u}_{2}(k)\right]
\end{gathered}
$$

The solutions of the difference equations (17) are

$$
\begin{gathered}
\mathbf{v}_{1}(k)=\mathbf{T}_{v_{0}}^{k} \mathbf{v}_{1}(0) \\
\mathbf{u}_{2}(k)=\mathbf{T}_{u_{0}}^{k} \mathbf{u}_{2}(0)+\sum_{j=0}^{k-1} \mathbf{T}_{u_{0}}^{j} \mathcal{T}_{u}\left[\mathbf{T}_{v_{0}}^{k-1-j} \mathbf{v}_{1}(0)\right]
\end{gathered}
$$
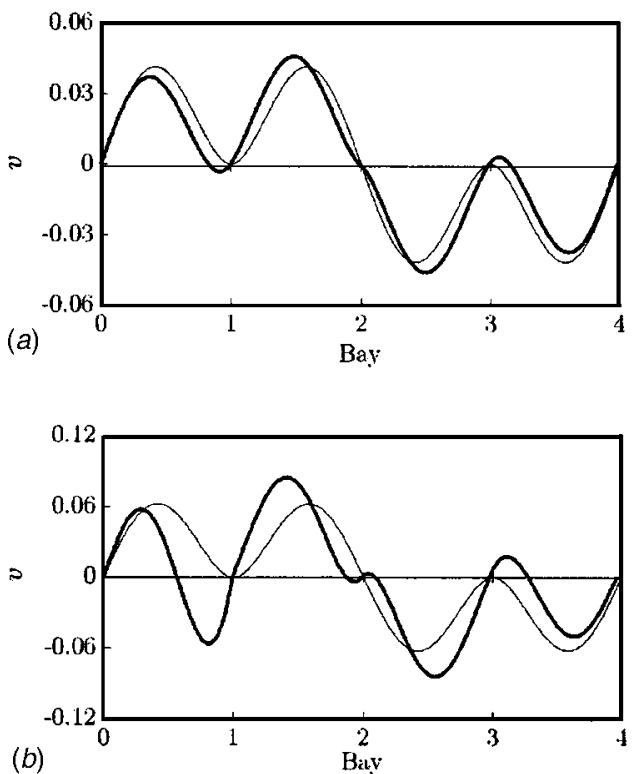

$$
\begin{gathered}
\mathbf{v}_{3}(k)=\mathbf{T}_{v_{0}}^{k} \mathbf{v}_{3}(0)+\sum_{j=0}^{k-1} \beta_{2} \mathbf{T}_{v_{0}}^{j} \mathbf{T}_{v_{0}}^{\prime} \mathbf{T}_{v_{0}}^{k-1-j} \mathbf{v}_{1}(0) \\
+\sum_{j=0}^{k-1} \mathbf{T}_{v_{0}}^{j} \mathcal{T}_{v}\left[\mathbf{T}_{v_{0}}^{k-1-j} \mathbf{v}_{1}(0), \mathbf{u}_{2}(k-1-j)\right]
\end{gathered}
$$

By introducing the global transfer matrix $\mathbf{S}_{v} \doteq \mathbf{T}_{v_{0}}^{N}$, the state at the end of the periodic beams is then obtained from Eq. (18) for $k$ $=N$

$$
\mathbf{v}_{1}(N)=\mathbf{S}_{v} \mathbf{v}_{1}(0)
$$
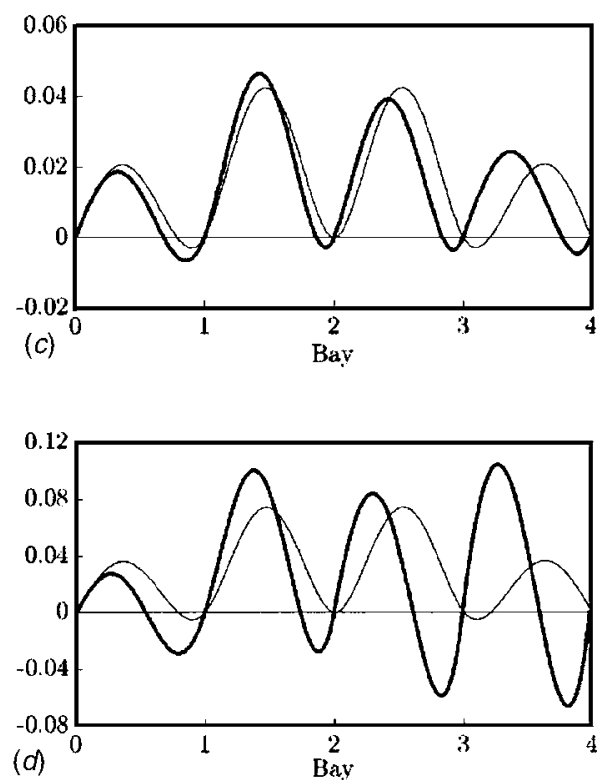

Fig. 6 Third and fourth mode of a 4-bay beam: (a) third mode $(\beta=3.926) a=0.04 \pi,(b)$ third mode $(\beta=3.926) a=0.06 \pi$, (c) fourth mode $(\beta=4.464) a=0.02 \pi$, and (d) fourth mode ( $\beta$ $=4.464) a=0.035 \pi ;$ thick lines, nonlinear; thin lines, linear 

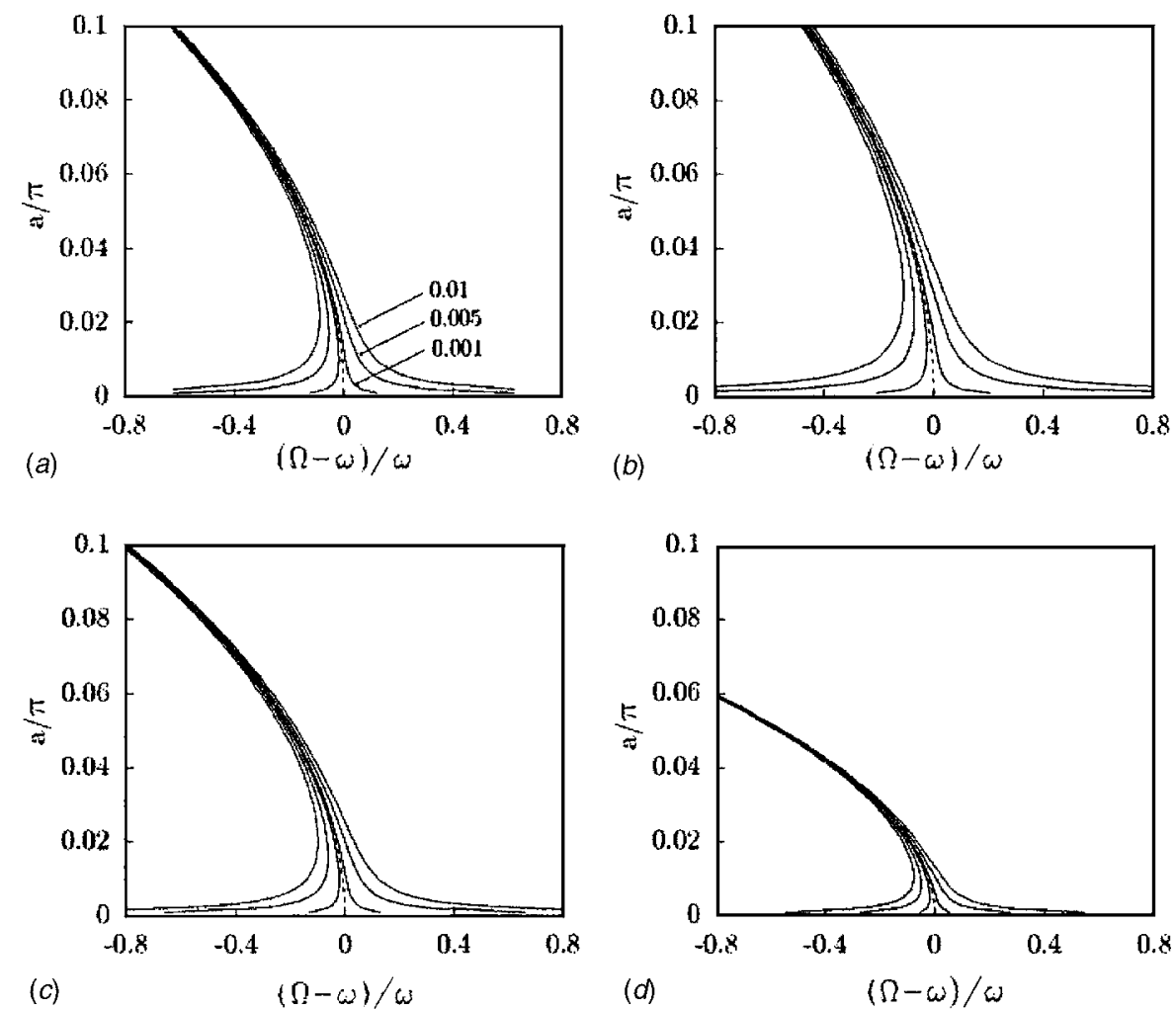

Fig. 7 Frequency-response relationship for a four-bay beam with $m_{0}$ $=0.0,0.001,0.005,0.01$ : (a) first mode, (b) second mode, (c) third mode, and (d) fourth mode $(\Omega, \omega$ : nonlinear and linear frequency)

$$
\begin{gathered}
\mathbf{u}_{2}(N)=\mathbf{S}_{u} \mathbf{u}_{2}(0)+\mathcal{S}_{u}\left[\mathbf{v}_{1}(0), \mathbf{0}\right] \\
\mathbf{v}_{3}(N)=\mathbf{S}_{v} \mathbf{v}_{3}(0)+\beta_{2} \mathbf{S}_{v}^{\prime} \mathbf{v}_{1}(0)+\mathcal{S}_{v}\left[\mathbf{v}_{1}(0), \mathbf{U}_{2}\right]
\end{gathered}
$$

where

$$
\begin{gathered}
\mathcal{S}_{\alpha}(\mathbf{x}, \mathbf{Y}) \doteq \sum_{j=0}^{N-1} \mathbf{T}_{\alpha 0}^{j} \mathcal{T}_{\alpha}\left[\mathbf{T}_{v_{0}}^{N-1-j} \mathbf{x}, \mathbf{y}(N-1-j)\right] \alpha=u, v \\
\mathbf{S}_{v}^{\prime} \doteq \sum_{j=0}^{N-1} \mathbf{T}_{v_{0}}^{j} \mathbf{T}_{v_{0}}^{\prime} \mathbf{T}_{v_{0}}^{N-1-j}
\end{gathered}
$$

with $\mathbf{Y}=[\mathbf{y}(0), \cdots, \mathbf{y}(N-1)]$

\section{Frequency-Amplitude Relationship}

An illustrative example consisting of a hinged-roll supported chain of beams loaded by a couple $m_{0}$ acting on the left-most support, is considered. The boundary conditions read

$$
\begin{gathered}
m(0)= \pm m_{0} \\
m(N)=0 \\
u(0)=0 \\
g(N)=0
\end{gathered}
$$

where the \pm in Eq. $(21 a)$ is due to the force being either in-phase or counterphase with the response. By using Eq. (16a) and (16b), the boundary conditions for the perturbation equation (19) follow:

$$
\begin{array}{cc}
\varepsilon: m_{1}(0)=m_{0}, & m_{1}(N)=0 \\
\varepsilon^{2}: u_{2}(0)=0, & g_{2}(N)=0
\end{array}
$$

$$
\varepsilon^{3}: m_{3}(0)=0, \quad m_{3}(N)=0
$$

5.1 Free Oscillations. The frequency-amplitude relation for free vibrations $\left(m_{0}=0\right)$ is sought. To define the amplitude of the modal shape, the normalization condition $\varphi(0)=\varepsilon \pi$ is chosen, with $\varepsilon$ being a scaling factor representing the midspan displacement to length ratio in the linear approximation. Consequently,

$$
\varphi_{1}(0)=\pi \text { and } \varphi_{3}(0)=0
$$

are the normalization conditions for the perturbation equations (19a) and (19c). The order $\epsilon$ equations (19a) read

$$
\epsilon:\left(\begin{array}{c}
\varphi_{1}(N) \\
m_{1}(N)
\end{array}\right)=\left[\begin{array}{ll}
S_{\varphi \varphi} & S_{\varphi m} \\
S_{m \varphi} & S_{m m}
\end{array}\right]\left(\begin{array}{c}
\varphi_{1}(0) \\
m_{1}(0)
\end{array}\right)
$$

From the second line of Equation (24), by accounting for the boundary condition $(22 a)$ and $(22 b)$

$$
S_{m \varphi}\left(\beta_{0}\right)=0
$$

provides with the roots $\beta_{0_{k}}$ corresponding to the natural frequencies of the small-amplitude oscillations. By iterating (18a), with $\mathbf{v}_{1}(0)=(\pi, 0)$, the linear modal shape is obtained.

The equations $(19 b)$ read

$$
\epsilon^{2}:\left(\begin{array}{l}
u_{2}(N) \\
g_{2}(N)
\end{array}\right)=\left[\begin{array}{ll}
S_{u u} & S_{u g} \\
S_{g u} & S_{g g}
\end{array}\right]\left(\begin{array}{l}
u_{2}(0) \\
g_{2}(0)
\end{array}\right)+\left(\begin{array}{l}
\mathcal{S}_{u}\left[\mathbf{v}_{1}(0), \mathbf{0}\right] \\
\mathcal{S}_{g}\left[\mathbf{v}_{1}(0), \mathbf{0}\right]
\end{array}\right)
$$

By taking into account the boundary condition $(22 b)$, the second line of Eq. (26) yields the axial force at the left end of the beams 


$$
g_{2}(0)=-\frac{\mathcal{S}_{g}\left[\mathbf{v}_{1}(0), \mathbf{0}\right]}{S_{g g}}
$$

consequently $\mathbf{u}_{2}(0)=\left[0, g_{2}(0)\right]$. By iterating Eq. $(18 b)$, starting from the known values of $\mathbf{u}_{2}(0)$ and $\mathbf{v}_{1}(0), \mathbf{u}_{2}(k)$ is evaluated at any node of the chain. Equations $(19 c)$ read

$$
\begin{aligned}
\epsilon^{3}:\left(\begin{array}{c}
\varphi_{3}(N) \\
m_{3}(N)
\end{array}\right)= & {\left[\begin{array}{ll}
S_{\varphi \varphi} & S_{\varphi m} \\
S_{m \varphi} & S_{m m}
\end{array}\right]\left(\begin{array}{c}
\varphi_{3}(0) \\
m_{3}(0)
\end{array}\right)+\beta_{2}\left[\begin{array}{cc}
S_{\varphi \varphi}^{\prime} & S_{\varphi m}^{\prime} \\
S_{m \varphi}^{\prime} & S_{m m}^{\prime}
\end{array}\right]\left(\begin{array}{c}
\varphi_{1}(0) \\
m_{1}(0)
\end{array}\right) } \\
& +\left(\begin{array}{l}
\mathcal{S}_{\varphi}\left[\mathbf{v}_{1}(0), \mathbf{U}_{2}\right] \\
\mathcal{S}_{m}\left[\mathbf{v}_{1}(0), \mathbf{U}_{2}\right]
\end{array}\right)
\end{aligned}
$$

From the second line of Eq. (28), by using the boundary conditions (22a) and (22c) and the normalization conditions (23), the natural frequency correction is derived as

$$
\beta_{2}=-\frac{\mathcal{S}_{m}\left[\mathbf{v}_{1}(0), \mathbf{U}_{2}\right]}{\pi S_{m \varphi}^{\prime}}
$$

Finally, by iterating $(18 c)$ with $\mathbf{v}_{3}(0)=(0,0)$, the nonlinear correction $\mathbf{v}_{3}(k)$ to the linear mode is obtained.

5.2 Forced Resonant Oscillations. In forced resonant motions the frequency $\beta=\beta_{0}+\epsilon^{2} \beta_{2}$ is prescribed, with $\beta_{2}$ representing a detuning parameter. At first, the boundary conditions (22a) are introduced in the second line of Eq. (24) to obtain the firstorder approximation of the left end rotation $\varphi_{1}(0)$. From Eq. $(18 a)$, the first approximation of the chain response is then obtained by using the boundary conditions $(22 b), g_{2}(0)$ is determined through the second line of Eq. (26). Once $\mathbf{u}_{2}(0)$ and $\mathbf{v}_{1}(0)$ are known, $\mathbf{u}_{2}(k)$ at any node of the chain is obtained by iterating Eq. (18b). From the second line of Eq. (28), the boundary conditions $(22 a)$ and $(22 c)$ and choosing $\varphi_{3}(0)=0$ as normalization condition, the frequency-amplitude relation follows:

$$
\beta_{2}=-\frac{\mathcal{S}_{m}\left[\mathbf{v}_{1}(0), \mathbf{U}_{2}\right]}{\varphi_{1}(0) S_{m \varphi}^{\prime} \pm m_{0} S_{m m}^{\prime}}
$$

\section{Numerical Results}

A chain of four simply supported beams, each of length $l$, with the left end hinged, is considered. The frequency-amplitude relation in (16) is evaluated by using either Eq. (29) and (30), for the free and forced case, respectively. The perturbation parameter $\epsilon$ entering equation in (16) is expressed either $\epsilon=a / \pi$ or $\epsilon$ $=a / \varphi_{1}(0)$ in the two cases, where $a \doteq \varphi(0)$ is the amplitude. The backbone curves of the free motion of the chain are first drawn. Figures 2 and 3 show these curves for the natural frequencies of the first four modes and for modes from the fifth to the eighth, respectively. The two sets of four curves have been shown separately as they belong to the first $(\pi<\beta<4.730)$ and second $(2 \pi$ $<\beta<7.853)$ pass band, respectively, of the corresponding linear model. As the amplitude of oscillations increases, all the natural frequencies trespass the lower frequency boundary of the corresponding linear pass band. The curves relevant to the third, fourth, seventh, and eighth modes are characterized by slopes higher than that of the neighboring ones, entailing crossings among several backbone curves. A softening type of behavior can be observed. This is due to the prevailing effect of the inertia nonlinearity with respect to the hardening effect of the elastic one [10]. Moreover, such a softening effect increases with the number of beams in the chain, as shown in Fig. 4 where the first (dimensional) natural frequency corrections versus the amplitude of the free oscillation are drawn for increasing number of beams entering the chain. Such a behavior is explained by the fact that each beam of the chain, in addition to the longitudinal displacements caused by the own transversal displacements, undergoes a longitudinal translation equal to the sum of the shortenings of all the preceding beams. Therefore, the longer the chain, the more important the

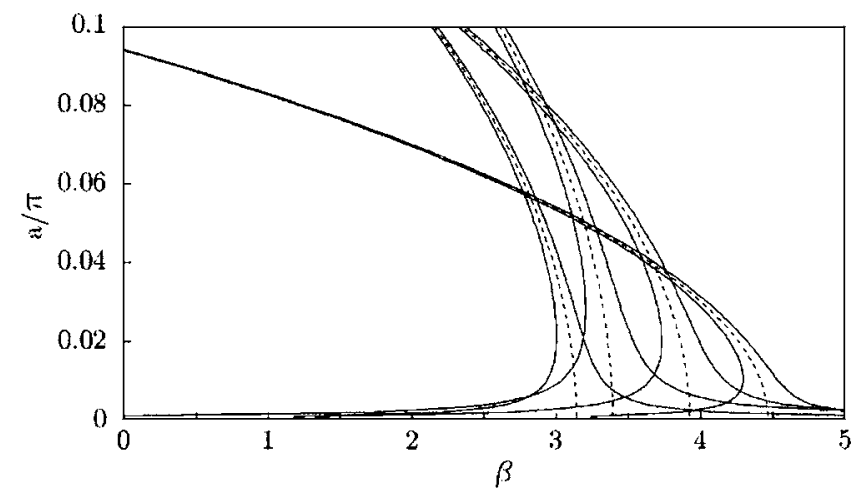

Fig. 8 Frequency-response relationship in the first linear pass band for a four-bay beam; $m_{0}=0.01$

longitudinal kinetic energy. The curve corresponding to the single beam coincides with the one obtained in [10], where the nonlinear dynamics of beams on movable supports have been studied. Figures 5 and 6 show the first four modal shapes; the comparison between the linear and nonlinear modes are depicted for increasing amplitude of oscillations. The nonlinear shortening effect destroys the symmetry of the linear modes; it can be best noted at the right end of the chain.

As far as the forced oscillations is concerned, Fig. 7 shows the frequency-response curves for the first four modes separately, for different intensities of the couple $m_{0}$. As shown in Fig. 8, where the frequency-response curves are drawn next to each other, such intensity must be limited in order for the present unimodal analysis to be meaningful.

\section{Conclusions}

The free and forced harmonic response of a chain of nonlinear beams resting on sliding supports has been determined. The periodicity of the system has been exploited by using an asymptotic approximation of the nonlinear transfer function. In analogy to the linear case, the approach leads to an algebraic set of equations whose dimensions are equal to the number of coupling degrees of freedom. Starting from the single inextensible and shear indeformable beam transfer function, the global transfer function has been derived in order to obtain the frequency-amplitude relationship. Because of the movable supports, the system is not symmetric with respect to longitudinal displacement and forces that enter the problem as second-order variables. For the beam model considered, it has been found that the softening effect due to the longitudinal inertia increases with the number of elements of the chain. Future investigations are in order to extend the procedure to multimodal analysis to assess the influence of closely spaced modes.

\section{Acknowledgment}

This work has been partially supported by the MIUR-COFIN 2001 grant.

\section{References}

[1] Sen Gupta, G., 1970, "Natural Flexural Waves and the Normal Modes of Periodically-Supported Beams and Plates," J. Sound Vib., 13, pp. 89-101.

[2] Faulkner, M. G., and Hong, D. P., 1985, "Free Vibrations of Mono-Coupled Periodic System," J. Sound Vib., 99, pp. 29-42. 
[3] Romeo, F., and Luongo, A., 2002, "Invariant Representation of Propagation Properties for Bi-coupled Periodic Structures," J. Sound Vib., 257, pp. 869886.

[4] Romeo, F., and Luongo, A., 2003, "Vibration Reduction in Piecewise Bicoupled Periodic Structures," J. Sound Vib., 268, pp. 601-615.

[5] Luongo, A., and Romeo, F., 2005, "Real Wave Vectors for Dynamic Analysis of Periodic Structures," J. Sound Vib. 279, pp. 309-325.

[6] Vakakis, A. F., and King, M. E., 1995, "Nonlinear Wave Transmission in a Mono-Coupled Elastic Periodic System," J. Acoust. Soc. Am., 98, pp. 15341546.

[7] Davies, M. A., and Moon, F. C., 1996, "Transition From Soliton to Chaotic
Motion Following Sudden Excitation of a Nonlinear Structure," ASME J. Appl. Mech., 63, pp. 445-449.

[8] Luongo, A., 1995, "A Transfer Matrix Perturbation Approach to the Buckling Analysis of Nonlinear Periodic Structures," 10th ASCE Conference, Boulder, pp. 505-508.

[9] Yong, Y., and Lin, Y. K., 1989, "Propagation of Decaying Waves in Periodic and Piecewise Periodic Structures of Finite Length," J. Sound Vib., 129, pp. 99-118.

[10] Luongo, A., Rega, G., and Vestroni, F., 1986, “On Nonlinear Dynamics of Planar Shear Indeformable Beams," ASME J. Appl. Mech., 53, pp. 619-624. 